\title{
Evolution of the transverse photoelectron-momentum distribution for atomic ionization driven by a laser pulse with varying ellipticity
}

\author{
I. A. Ivanov* \\ Research School of Physical Sciences, The Australian National University, Canberra ACT 0200, Australia
}

(Received 2 May 2014; published 22 July 2014)

\begin{abstract}
We consider the process of atomic ionization driven by a laser pulse with varying ellipticity. We study the distribution of the momenta of the photoelectrons, ionized by a strong laser field, emitted in the direction perpendicular to the polarization plane (transverse distribution). We show that with changing laser pulse ellipticity, the transverse distribution evolves from the singular cusplike distribution for the close to linear polarization to the smooth Gaussian-like structure for the close to circular polarization. In the latter case, when the ellipticity parameter $\epsilon \rightarrow 1$, the strong-field approximation formula for the transverse momentum distribution becomes quantitatively correct.
\end{abstract}

DOI: 10.1103/PhysRevA.90.013418

PACS number(s): $32.80 . \mathrm{Rm}, 32.80 . \mathrm{Fb}, 42.50 . \mathrm{Hz}$

\section{INTRODUCTION}

Tunneling theories of photoionization proved to be in the past (and still are) of great importance in understanding atomic or molecular ionization by a strong IR laser field. By tunneling theories we mean here a broad class of theories comprising original work on the strong-field approximation (SFA) by Keldysh [1], its modification, known as the Keldysh-FaisalReiss (KFR) theory [2,3], subsequent developments [4-9], and quasistatic theories exploiting the fact that the IR field varies slowly in time [10,11].

The SFA in its original form [1] did not include any interaction of the ejected electron and parental ion. The importance of the effect of the Coulomb field of the parental ion on the ionization process has, however, long been realized. The Coulomb field of the residual ion was found to be responsible for such effects as low-energy structures in electron spectra [12] or two-dimensional momentum distributions [13], Coulomb focusing [14], asymmetry in the spectra of abovethreshold ionization spectra [15], and the formation of a cusp in the transverse electron-momentum distribution [16].

It is the latter effect, the influence of the Coulomb field of the parental ion on the transverse (i.e., perpendicular to the polarization plane of the driving pulse) electron-momentum distribution, which will interest us below. Studying the electron-momentum distributions (in both longitudinal and transverse directions) can shed light on the fine details of the strong-field ionization process $[9,17]$.

It has been found [16] that for the case of the linearly polarized laser pulse, the transverse electron-momentum distribution exhibits a sharp cusplike peak at zero transverse momentum. For the case of the circularly polarized light, on the contrary, this distribution was found not to deviate considerably from the Gaussian-like structure predicted by the SFA [18]. We see thus two different forms (cusplike and Gaussian-like) of the transverse electron-momentum distribution for two limiting polarization states of the driving pulse. The aim of the present work is to study the transition between these two forms in detail. We shall consider below an atom in the field of an elliptically polarized laser pulse and shall

*Igor.Ivanov@anu.edu.au consider the evolution of the transverse electron-momentum distribution with varying ellipticity. We use the hydrogen atom as a model; atomic units are used throughout the paper.

\section{THEORY}

We solve the time-dependent Schrödinger equation (TDSE) for a hydrogen atom:

$$
i \frac{\partial \Psi(\boldsymbol{r})}{\partial t}=\left[\hat{H}_{\mathrm{atom}}+\hat{H}_{\mathrm{int}}(t)\right] \Psi(\boldsymbol{r}) .
$$

The operator $\hat{H}_{\text {int }}(t)$ in Eq. (1) describes the interaction of the atom with the electromagnetic field. We use the velocity form for this operator:

$$
\hat{H}_{\mathrm{int}}(t)=\boldsymbol{A}(t) \cdot \hat{\boldsymbol{p}}
$$

with

$$
\boldsymbol{A}(t)=-\int_{0}^{t} \boldsymbol{E}(\tau) d \tau
$$

The laser pulse is elliptically polarized (with the ellipticity parameter $\epsilon$ ) and propagates along the $z$ direction (assumed to be the quantization axis). Its field components are

$$
E_{x}=\frac{E}{\sqrt{1+\epsilon^{2}}} f(t) \cos \omega t, E_{y}=\frac{E \epsilon}{\sqrt{1+\epsilon^{2}}} f(t) \sin \omega t .
$$

In the calculations presented below we use $E=0.0534$ a.u., which corresponds to an intensity of $10^{14} \mathrm{~W} / \mathrm{cm}^{2}$ for the laser pulse described by Eq. (4). For the base frequency of the pulse we use $\omega=0.057$ a.u. (corresponding to a wavelength of $790 \mathrm{~nm}$ ). In Eq. (4) the function $f(t)=\sin ^{2}\left(\pi t / T_{1}\right)$ (here $T_{1}$ is the total pulse duration) is a pulse envelope. We report below results for the pulse durations $T_{1}=4 T$ and $T_{1}=10 T$, where $T=2 \pi / \omega$ is an optical cycle of the field (4).

The initial state of the system is the hydrogen ground state. To solve the TDSE we employ the strategy used in Refs. [19,20].

The solution of the TDSE is represented as a partial-wave series:

$$
\Psi(\boldsymbol{r}, t)=\sum_{l=0}^{L_{\max }} \sum_{\mu=-l}^{l} f_{l \mu}(r, t) Y_{l \mu}(\theta, \phi)
$$


The radial part of the TDSE is discretized on a grid with a step size $\delta r=0.1$ a.u. in a box with size $R_{\max }=400$ a.u. for a total pulse duration of four optical cycles and $R_{\max }=1000$ a.u. for a pulse duration of ten optical cycles. In the calculations we used $L_{\max }=50$ in Eq. (5). A series of routine checks ensuring that the calculation is well converged with respect to variations of the parameters $\delta r, R_{\max }$, and $L_{\max }$ has been performed.

Substituting the expansion (5) into the TDSE gives us a system of coupled equations for the radial functions $f_{l \mu}(r, t)$, describing the evolution of the system in time. To solve this system we use the matrix iteration method developed in [21]. Ionization amplitudes $a(\boldsymbol{p})$ are obtained by projecting the solution of the TDSE at the end of the laser pulse on the set of the ingoing scattering states $\psi_{p}^{(-)}(\boldsymbol{r})$ of hydrogen atom.

In the present paper we are interested in the transverse electron-momentum distribution, describing the probability of detecting a photoelectron with a given value of the momentum component $p_{\perp}$ perpendicular to the polarization plane. For the geometry we use $p_{\perp}=p_{z}$. The transverse momentum distribution can be obtained therefore as

$$
W\left(p_{\perp}\right)=\int|a(\boldsymbol{p})|^{2} d p_{x} d p_{y} .
$$

The well-known SFA result for the transverse momentum distribution in the case of the elliptically polarized laser pulse is a smooth Gaussian form [5]:

$$
W\left(p_{\perp}\right) \propto \exp \left\{-\frac{(2 I)^{1 / 2} \sqrt{1+\epsilon^{2}}}{E} p_{\perp}^{2}\right\},
$$

where $I$ is the ionization potential, and we employ the notation used in Eq. (4) for the laser field parameters.

This smooth distribution is in striking contrast to the cusplike structure observed in the experiment [16] for the ionization of noble gases by a linearly polarized laser field [which corresponds to the case of $\epsilon=0$ in Eq. (7)]. This cusplike structure has been attributed to the Coulomb effects, which are, of course, neglected in the SFA.

In the next section we shall present the results of a systematic study of the perpendicular momentum distributions for various values of the ellipticity parameter $\epsilon$. We shall see that when the ellipticity parameter varies between $\epsilon=0$ (linear polarization) and $\epsilon=1$ (circular polarization), spectra evolve from the cusplike spectra observed in [16] to the Gaussian form predicted by the SFA and observed in [18].

\section{RESULTS}

Figure 1 shows the evolution of the distribution $W\left(p_{\perp}\right)$ with varying ellipticity parameter $\epsilon$ when ionization is driven by a short laser pulse with the duration $T_{1}=4 T$ (four optical cycles). A feature of the spectra clearly seen from Fig. 1 is the rapid decline of the transverse distribution with the ellipticity parameter $\epsilon$. This decline agrees with Eq. (7), which predicts exponential dependence of the transverse distribution on the ellipticity parameter for a given field strength, which has the same value, $E=0.0534$ a.u., for all values of $\epsilon$. Another feature clearly seen in Fig. 1 is the gradual transition from the cusplike structure in the spectra to the smooth Gaussian form. As one can see, the cusplike structure survives until $\epsilon \approx 0.5$.
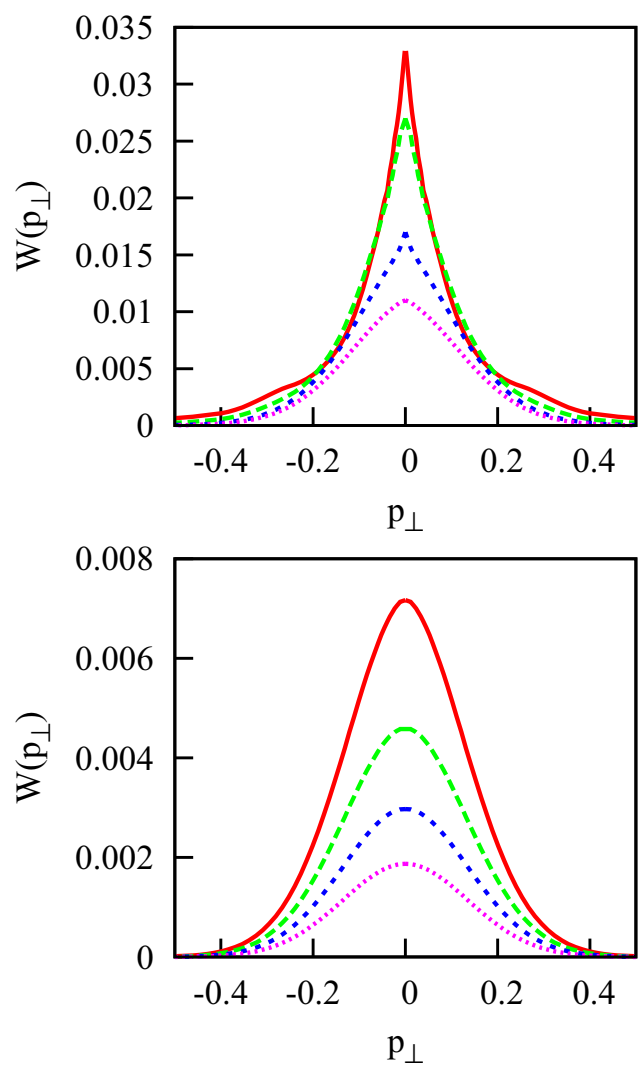

FIG. 1. (Color online) (top) Distribution $W\left(p_{\perp}\right)$ for $\epsilon=0$ (solid red line), $\epsilon=0.2$ (long-dashed green line), $\epsilon=0.4$ (short-dashed blue line), and $\epsilon=0.5$ (dotted magenta line). (bottom) Distribution $W\left(p_{\perp}\right)$ for $\epsilon=0.6$ (solid red line), $\epsilon=0.7$ (long-dashed green line), $\epsilon=0.8$ (short-dashed blue line), and $\epsilon=1$ (dotted magenta line). Pulse parameters are $E=0.0534$ a.u., $\omega=0.057$ a.u., and a pulse duration of four optical cycles.

To gain better insight into this interesting phenomenon we show in Figs. 2 and 3 the function $V\left(p_{\perp}\right)=\log W\left(p_{\perp}\right)$ for a narrow interval in the vicinity of the singular point $p_{\perp}=0$. For the distribution $W\left(p_{\perp}\right)$ to have a cusp at $p_{\perp}=0, V\left(p_{\perp}\right)$ must clearly be singular there. The nature of this singularity can be guessed from the character of the singularity of $W\left(p_{\perp}\right)$ seen in Fig. 1 for small values of the ellipticity parameter $\epsilon$. One can see that $W\left(p_{\perp}\right)$ is continuous at $p_{\perp}=0$ but may have infinite derivatives there. Clearly, $V\left(p_{\perp}\right)$ must possess the same properties. It is natural therefore to suggest that in the vicinity of the singular point $p_{\perp}=0$ function $V\left(p_{\perp}\right)$ can be represented as

$$
V\left(p_{\perp}\right)=B+A\left|p_{\perp}\right|^{\alpha} .
$$

We may look at this equation as a tentative expression for the first two terms of an expansion of $V\left(p_{\perp}\right)$ in the vicinity of the singular point. We perform next a series of least-squares fits, fitting the function $V\left(p_{\perp}\right)$ using Eq. (8) and considering the coefficients $A, B, \alpha$ in this equation as fitting parameters.

Results are shown in Figs. 2 and 3. One can see that Eq. (8) approximates the function $V\left(p_{\perp}\right)$ in the vicinity of the singular point fairly accurately. The quality of the fit does not depend appreciably on the interval of data we use as an input for the fitting procedure as long as this interval is sufficiently 

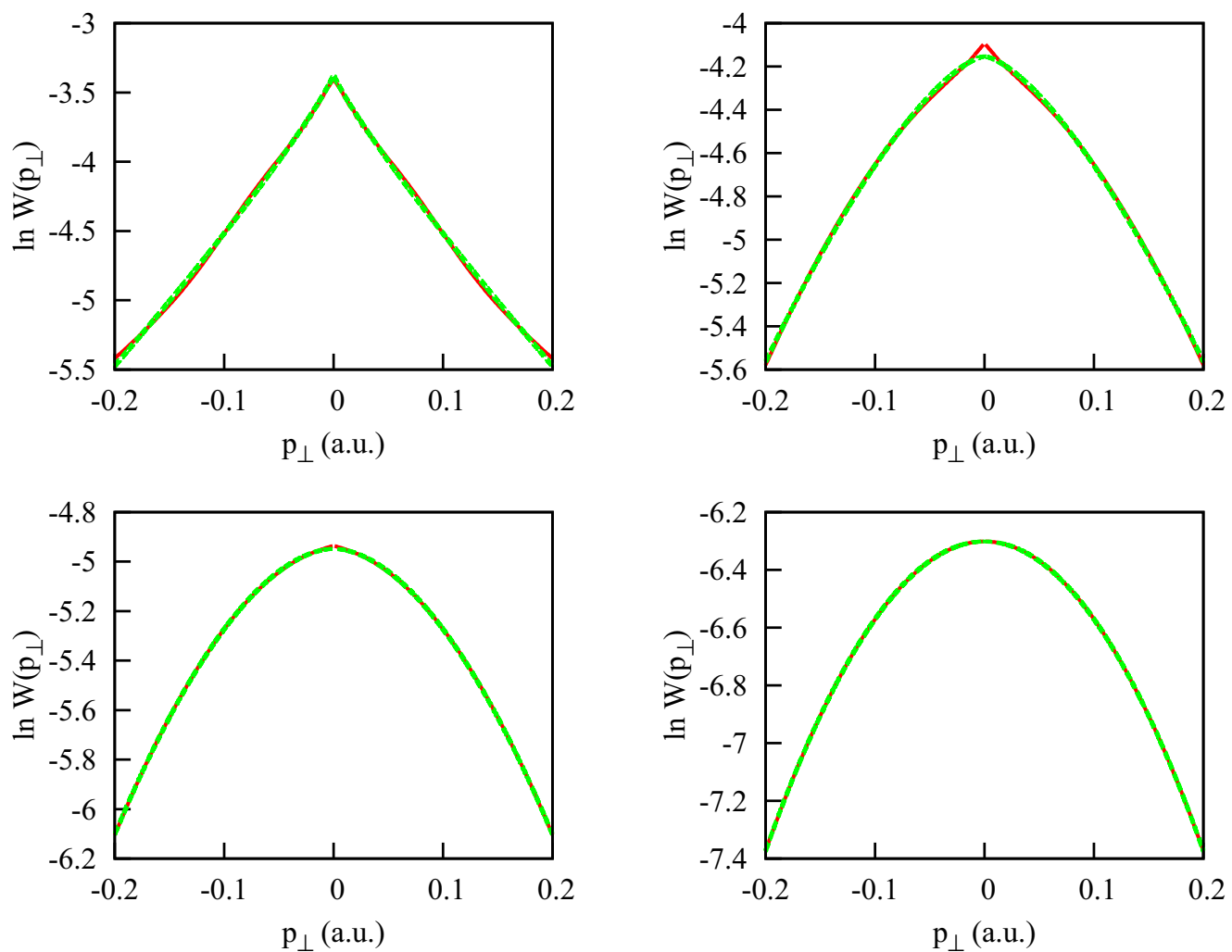

FIG. 2. (Color online) Function $V\left(p_{\perp}\right)=\ln W\left(p_{\perp}\right)$ (red solid line) and results of the fit based on Eq. (8) for $\epsilon=0,0.4,0.6,1$ (left to right, top to bottom). Pulse parameters are $E=0.0534$ a.u., $\omega=0.057$ a.u., and a pulse duration of four optical cycles.
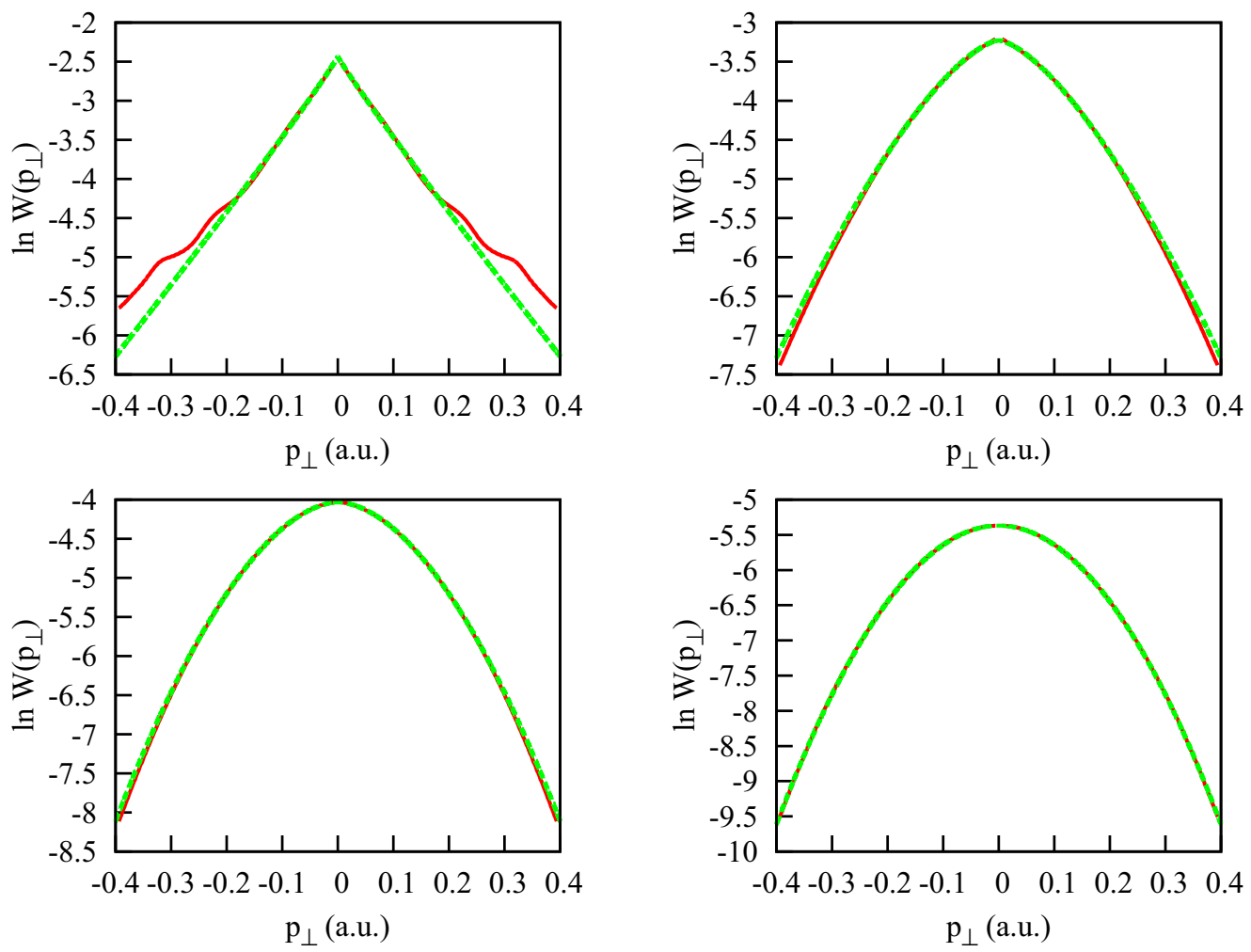

FIG. 3. (Color online) Function $V\left(p_{\perp}\right)=\ln W\left(p_{\perp}\right)$ (red solid line) and results of the fit based on Eq. (8) for $\epsilon=0,0.4,0.6,1$ (left to right, top to bottom). Pulse parameters are $E=0.0534$ a.u., $\omega=0.057$ a.u., and a pulse duration of ten optical cycles. 

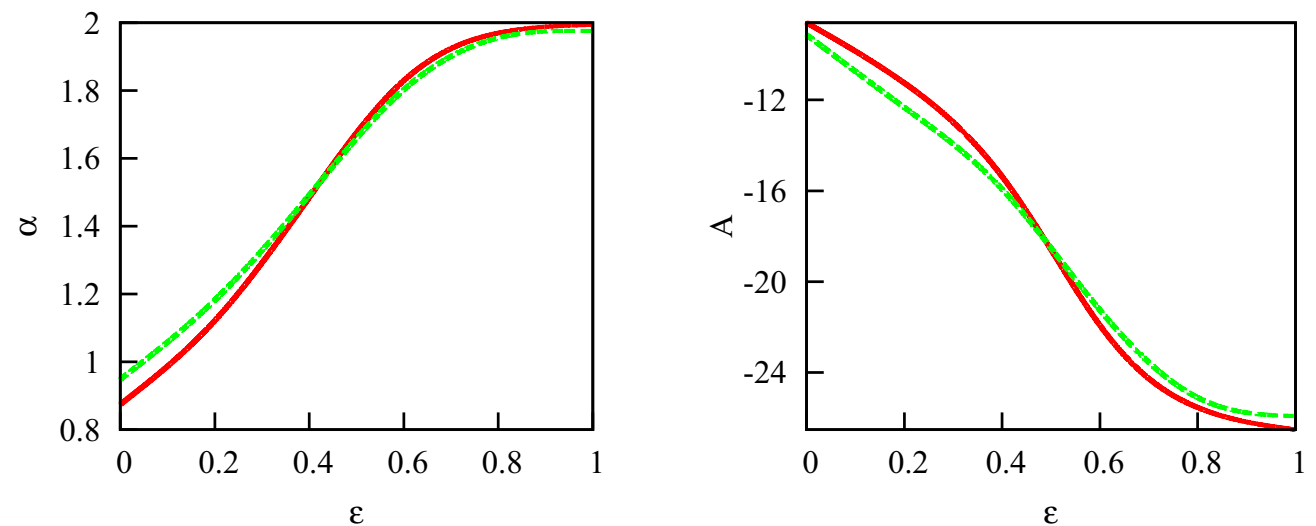

FIG. 4. (Color online) Fitting parameters $A$ and $\alpha$ in Eq. (8) as functions of the ellipticity parameter $\epsilon$. The pulse duration is four optical cycles (red solid line) and ten optical cycles (green dashed line). Other pulse parameters are $E=0.0534$ a.u., $\omega=0.057$ a.u.

narrow [results shown in Figs. 2 and 3 have been obtained using the interval $p_{\perp} \in(-0.2,0.2)$ for the fitting procedure]. The accuracy of the fit shown in Figs. 2 and 3 makes the conjecture that Eq. (8) indeed describes faithfully the behavior of $V\left(p_{\perp}\right)$ in the vicinity of the singular point plausible enough. Further support in favor of the validity of this conjecture is found on closer inspection of the coefficients of the fitting formula (8), which the fitting procedure gave us.

Fitting parameters $A$ and $\alpha$, which describe the character of the singular point, are of the most interest to us. Their dependence on the ellipticity parameter $\epsilon$ is shown in Fig. 4. For the nearly circular polarization parameter $\alpha \approx 2$, as prescribed by the tunneling equation (7). Moreover, parameter $A$, as predicted by the tunneling equation, should approach the value $A=-26.48$ for $\epsilon=1$. We see thus that for the nearly circular polarization the tunneling equation (7) describes the electron spectrum fairly accurately. With decreasing values of the ellipticity parameter the deviation from the Gaussian-like behavior due to the Coulomb effects becomes more and more significant.

This behavior can be understood if we take into account that the angular momentum composition of the wave function changes considerably with the ellipticity parameter. If we rewrite Eq. (5) as a sum of $\Psi_{l}(\boldsymbol{r}, t)$, where each $\Psi_{l}(\boldsymbol{r}, t)$ includes only spherical harmonics of rank $l$, then distribution

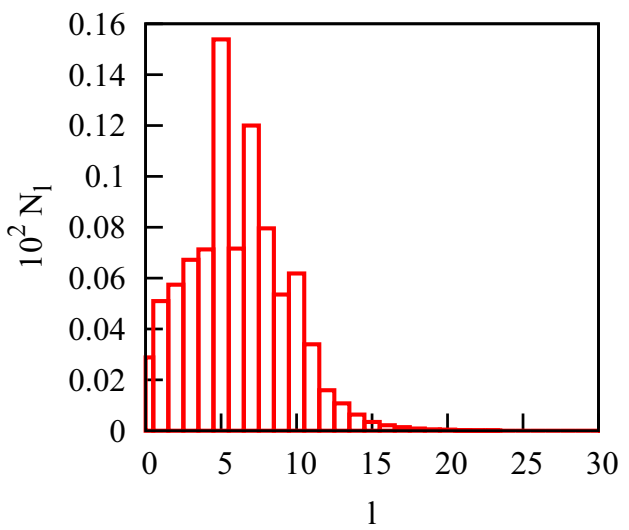

of the squared norms $N_{l}=\left|\Psi_{l}\right|^{2}$ can be used to characterize the angular momentum composition of the wave function. Since absorption of a photon from the circularly polarized wave increases the magnetic quantum number by one unit, we can expect that this distribution will be shifted toward larger $l$ for a pulse polarization approaching a circular one. That reasoning is illustrated in Fig. 5, where distributions of the norms $N_{l}$ for the wave function after the end of the laser pulse are shown for the cases of $\epsilon=0$ and $\epsilon=0.8$. We subtracted the contribution of the ground state to the $l=0$ component. The expected shift towards larger $l$ values is clearly seen in the Fig. 5. This shift towards larger angular momenta means much a higher centrifugal barrier and, consequently, larger electron-parental-ion separation for the close to circular polarization, which should diminish the role of the Coulomb effects.

The role of the high angular momenta can be further clarified if we consider transverse distributions obtained by setting in Eq. (5) all components of the wave function with angular momenta less than a certain value $L_{\min }$ to zero. This means that projecting the solution of the TDSE on the ingoing scattering states of the hydrogen atom, we ignore all Coulomb continuum wave functions with $l<L_{\min }$.

Figure 6 shows the transverse distributions computed for different values of the parameter $L_{\min }$ for the case of the

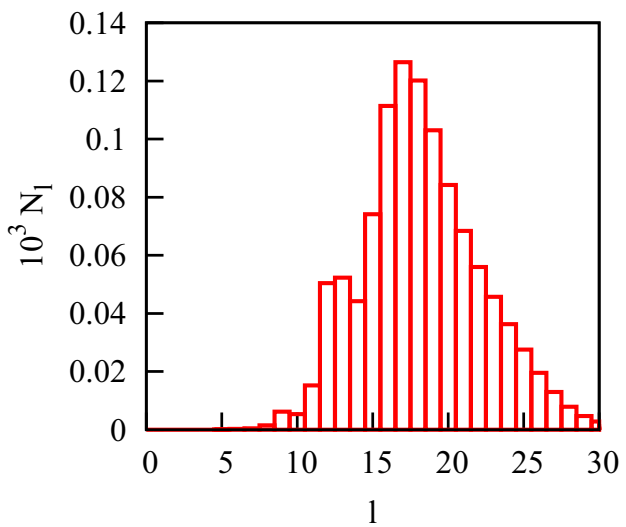

FIG. 5. (Color online) Angular momentum distributions for (left) $\epsilon=0$ and (right) $\epsilon=0.8$. Pulse parameters are $E=0.0534$ a.u., $\omega=$ 0.057 a.u., and a pulse duration of four optical cycles. 


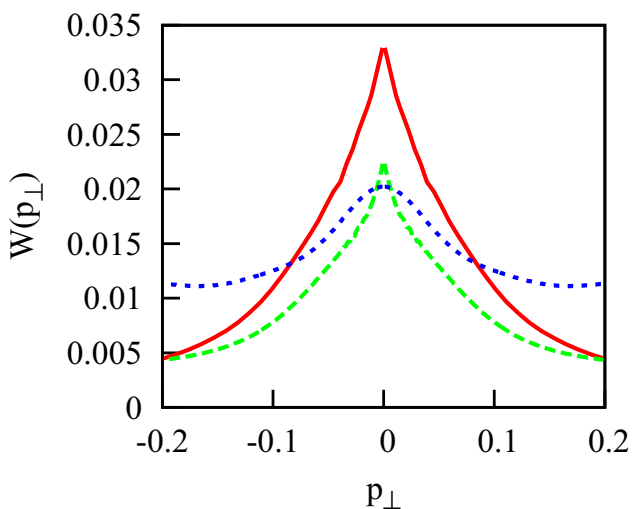

FIG. 6. (Color online) Distribution $W\left(p_{\perp}\right)$. Projection on the Coulomb scattering states with $L_{\min }=0$ (solid red line), $L_{\min }=5$ (long-dashed green line), $L_{\min }=10$ (short-dashed blue line). The distribution for $L_{\min }=10$ is scaled by a factor of 10 for better visibility. Pulse parameters are $\epsilon=0, E=0.0534$ a.u., $\omega=0.057$ a.u., and a pulse duration of four optical cycles.

linearly polarized laser pulse. One can see that if we leave in the wave function only the components with angular momenta $l \geqslant 10$ the cusp structure disappears, and we obtain a Gaussian transverse distribution. It was suggested in [16] that for the case of the linear polarization, the cusp originates from the singularity of the Coulomb continuum wave function at zero energy. The absence of the cusp in Fig. 6 for $L_{\text {min }}=10$ suggests that if we use for the projection operation only the Coulomb continuum wave functions with high angular momenta, this singularity somehow disappears or at least becomes less visible.

A plausible explanation for this fact can be obtained if we consider in more detail the projection operation we perform to find the ionization probabilities. This projection operation, as we mentioned above, relies on the calculation of the overlap integrals between the TDSE solution and the Coulomb continuum wave functions. The Coulomb continuum wave function is an object notoriously singular at the point $E=0$. Overlap integrals naturally inherit this singularity, which, as was suggested in [16], produces a cusp in the momentum distribution.

The character of the singularity inherited from the Coulomb continuum wave functions may, however, be different for different angular momenta $l$. One can put forward arguments showing that this singularity becomes milder with increasing $l$. The well-known small- $r$ expression for the radial Coulomb continuum wave function is (we use energy normalization here) [22]

$$
R_{E l}(r) \approx \frac{2^{l+1}}{(2 l+1) !} r^{l+1} \quad r \rightarrow 0, \quad E \rightarrow 0 .
$$

This expression, considered as a function of energy, is, of course, nonsingular. The TDSE solution we obtain, considered as a function of spatial variables, has some finite spatial extension $a$. It is a known property of the Coulomb wave functions [23] that for any $a$ the small- $r$ asymptotic expression (9) faithfully reproduces $R_{E l}(a)$ if the angular momentum $l$ is high enough. For such $l$, substituting the asymptotic form (9) into the overlap integral is legitimate, and we obtain a result which is nonsingular in energy. We can see therefore that the overlap integrals between the TDSE solution and the continuum Coulomb wave functions become less and less singular with increasing $l$. This, we believe, is the mechanism which effectively removes the singularity in the overlap integrals and hence in the momentum distributions if only high angular momenta are important in the expansion (5) of the wave function. This, in particular, is the case of atomic ionization by a laser pulse with close to circular polarization.

\section{CONCLUSION}

We studied the evolution of the transverse momentum distribution $W\left(p_{\perp}\right)$, describing the distribution of electron momenta in the direction perpendicular to the polarization plane, with the change of the ellipticity parameter. We saw the gradual change of the character of this distribution from the singular cusplike distribution for the close to linear polarization to the smooth Gaussian-like structure for the close to circular polarization state.

In the latter case, when the ellipticity parameter $\epsilon \rightarrow 1$, we see that the tunneling formula (7) becomes quantitatively correct. This does not necessarily mean that Coulomb effects are completely absent for the circular polarization. It is hardly possible, indeed, that a singular point of a generic function depending on a parameter (the ellipticity parameter in the present case) can disappear completely when the parameter gradually changes. We would rather be inclined to believe that the singularity simply becomes much more difficult to see. Indeed, Fig. 4 shows that exponent $\alpha$ in Eq. (8) is less than 1 for $\epsilon=0$. This means that $W\left(p_{\perp}\right)$ has an infinite first derivative at $p_{\perp}=0$. At the value of the ellipticity parameter $\epsilon \approx 0.1$, the first derivative of $W\left(p_{\perp}\right)$ ceases to be infinite at $p_{\perp}=0$. It is the second derivative of $W\left(p_{\perp}\right)$ which becomes infinite at this point. The singularity therefore becomes milder but does not go away completely. We believe this is what might happen for $\epsilon=1$; some higher-order derivatives of $W\left(p_{\perp}\right)$ at the point $p_{\perp}=0$ are still infinite, which is, of course, very difficult to establish in numerical calculations.

From a practical point of view, the Coulomb effects in the distribution $W\left(p_{\perp}\right)$ of the electron momenta in the direction perpendicular to the polarization plane become hardly important when the ellipticity parameter is as large as 0.8. As we have seen, the tunneling formula (7) works quite reliably for such a degree of polarization. The reason for this diminishing of the role of the Coulomb effects can be attributed, we believe, to the mechanism which we described above, which effectively removes the singularity from the overlap integrals for the close to circular polarization of the laser pulse.

\section{ACKNOWLEDGMENT}

The author acknowledges support from the Australian Research Council in the form of Discovery Grant No. DP120101805. The author is grateful to Professor A. S. Kheifets for the stimulating discussions and to a referee for valuable suggestions. The resources of the National Computational Infrastructure (NCI) Facility were employed. 
[1] L. V. Keldysh, Sov. Phys. JETP 20, 1307 (1965).

[2] F. H. M. Faisal, J. Phys. B 6, L89 (1973).

[3] H. R. Reiss, Phys. Rev. A 22, 1786 (1980).

[4] A. M. Perelomov and V. S. Popov, Sov. Phys. JETP 25, 482 (1967).

[5] V. S. Popov, Phys. Usp. 47, 855 (2004).

[6] A. Becker and F. H. M. Faisal, J. Phys. B 38, R1 (2005).

[7] F. H. M. Faisal and G. Schlegel, J. Mod. Opt. 53, 207 (2006).

[8] D. G. Arbó, J. E. Miraglia, M. S. Gravielle, K. Schiessl, E. Persson, and J. Burgdörfer, Phys. Rev. A 77, 013401 (2008).

[9] I. Dreissigacker and M. Lein, Chem. Phys. 414, 69 (2013).

[10] N. B. Delone and V. P. Krainov, J. Opt. Soc. Am. B 8, 1207 (1991).

[11] M. V. Ammosov, N. B. Delone, and V. P. Krainov, Sov. Phys. JETP 64, 1191 (1986).

[12] C. I. Blaga, F. Catoire, P. Colosimo, G. G. Paulus, H. G. Muller, P. Agostini, and L. F. DiMauro, Nat. Phys. 5, 335 (2008).

[13] Z. Chen, T. Morishita, A.-T. Le, M. Wickenhauser, X. M. Tong, and C. D. Lin, Phys. Rev. A 74, 053405 (2006).
[14] T. Brabec, M. Y. Ivanov, and P. B. Corkum, Phys. Rev. A 54, R2551 (1996).

[15] S. P. Goreslavski, G. G. Paulus, S. V. Popruzhenko, and N. I. Shvetsov-Shilovski, Phys. Rev. Lett. 93, 233002 (2004).

[16] A. Rudenko, K. Zrost, T. Ergler, A. B. Voitkiv, B. Najjari, V. L. B. de Jesus, B. Feuerstein, C. D. Schröter, R. Moshammer, and J. Ullrich, J. Phys. B 38, L191 (2005).

[17] A. N. Pfeiffer, C. Cirelli, A. S. Landsman, M. Smolarski, D. Dimitrovski, L. B. Madsen, and U. Keller, Phys. Rev. Lett. 109, 083002 (2012).

[18] L. Arissian, C. Smeenk, F. Turner, C. Trallero, A. V. Sokolov, D. M. Villeneuve, A. Staudte, and P. B. Corkum, Phys. Rev. Lett. 105, 133002 (2010).

[19] I. A. Ivanov, Phys. Rev. A 83, 023421 (2011).

[20] I. A. Ivanov and A. S. Kheifets, Phys. Rev. A 87, 033407 (2013).

[21] M. Nurhuda and F. H. M. Faisal, Phys. Rev. A 60, 3125 (1999).

[22] L. D. Landau and E. M. Lifshitz, Quantum Mechanics (Pergamon Press, Oxford, New York, 1965).

[23] M. Abramowitz and I. E. Stegun, Handbook of Mathematical Functions (National Bureau of Standards, Washington, D.C., 1967). 